\title{
Accuracy of five intraocular lens formulas in eyes with trifocal lens implant
}

\author{
MONICA MĂLĂESCU ${ }^{1}$, HORIA T. STANCA ${ }^{2}$, BOGDANA TĂBĂCARU ${ }^{2}$, \\ ADRIANA STĂNILA $\breve{A}^{1}$, SIMONA STANCA ${ }^{3}$ and CIPRIAN DANIELESCU ${ }^{4}$ \\ ${ }^{1}$ Department of Ophthalmology, Faculty of Medicine, 'Lucian Blaga' University, 550159 Sibiu; \\ Departments of ${ }^{2}$ Ophthalmology and ${ }^{3}$ Pediatrics, 'Carol Davila' University of Medicine \\ and Pharmacy, 050474 Bucharest; ${ }^{4}$ Department of Ophthalmology, 'Grigore T. Popa' \\ University of Medicine and Pharmacy, 700115 Iasi, Romania
}

Received April 22, 2020; Accepted May 22, 2020

DOI: $10.3892 / \mathrm{etm} .2020 .8891$

\begin{abstract}
Accuracy of intraocular lens (IOL) calculation formulas SRK/T, Hoffer Q, Holladay 1, Haigis and Barrett Universal II were compared in prediction of postoperative refraction for multifocal and implants using a single optical biometry device. The authors included 88 refractive lens exchange and cataract surgeries, with AcrySof IQ PanOptix implant (Alcon Laboratories, Inc.). All eyes were divided into three groups based on axial length (AL), group 1: $<22 \mathrm{~mm}$ (14 eyes), group 2: 22-24.5 mm (68 eyes) and group 3: $>24.5 \mathrm{~mm}$ (6 eyes). The refractive prediction error (RPE) and mean absolute error (MAE) were calculated for 5 different formulas: SRK/T, Hoffer Q, Holladay 1, Haigis and Barrett Universal II. For eyes with the AL between $22 \mathrm{~mm}$ and $24.5 \mathrm{~mm}$ the greatest percentage of eyes with RPEs within $\pm 0.25 \mathrm{D}$ was $32.4 \%$ for Haigis formula, followed by Barrett Universal II, Hoffer Q and Holladay 1 with $29.4 \%$. The percentage of eyes with RPEs within $\pm 0.50 \mathrm{D}$ was $100 \%$ only for Barrett Universal II and Holladay 1, 94.1\% for SRK/T and $91.2 \%$ for Haigis and Hoffer Q. The first and third group with $\mathrm{AL}<22$ and $>24.5 \mathrm{~mm}$ were too small to have statistical significance due to the reluctancy to use multifocal IOLs on extreme ALs. ANOVA test showed no statistical difference $(\mathrm{P}=0.166)$ between the RPEs measured for each formula in this cohort. This study showed no statistical difference between formulas for this trifocal lens implant. There was a tendency for the RPE to be within $\pm 0.25 \mathrm{D}$ for most of the eyes with the Haigis formula, and within $\pm 0.50 \mathrm{D}$ for all the eyes with the Barrett Universal II formula in the group with the AL between 22 and $24.5 \mathrm{~mm}$.
\end{abstract}

Correspondence to: Dr Horia T. Stanca, Department of Ophthalmology, 'Carol Davila' University of Medicine and Pharmacy, 8 Eroilor Sanitari Street, 050474 Bucharest, Romania

E-mail: hstanca@yahoo.com

Key words: hydrophobic, intraocular lens formulas, multifocal, optical biometry, PanOptix, presbyopia, trifocal

\section{Introduction}

With the progress of medical technology and better intraocular lens (IOL) quality, modern biometry with incorporated 4th generation formulas refractive outcome prediction accuracy has increased (1-3). Therewith, refractive expectations following cataract surgery are rising. More patients request refractive lens exchange hoping they can be free of spectacles, even for presbyopia. The demand and use of multifocal IOLs have risen and, thus, the need for modern calculation formulas with great refractive outcome prediction has increased as well.

AcrySof IQ PanOptix is a non-apodized diffractive hydrophobic monoblock IOL with an ultraviolet filter and a blue light filter (4). It has a $6.0 \mathrm{~mm}$ optical zone, consisting of a $4.5 \mathrm{~mm}$ central trifocal region with 15 diffraction rings and an external refractive rim $(5,6)$. The 3 foci are for distance vision, for intermediary vision with an addition of $+2.17 \mathrm{D}$, and for near vision with an addition of $+3.25 \mathrm{D}(7,8)$. The preferred focal point is at a distance of $60 \mathrm{~cm}(4,7)$. The light distribution within the lens is as follows: $50 \%$ for the distance vision, $25 \%$ for intermediary vision and $25 \%$ for near vision (9).

In Romania, older generation formulas like SRK/T, Hoffer Q and Holladay 1 are still popular, because ultrasound biometers, which include them, are still used. However, more modern formulas with greater accuracy are now available with the new optical biometers (1-3,7). Most of these 4th generation formulas are incorporated into a biometer software and have become more accessible. Calculation software and online calculators are also available, and most are free for use, but they are more prone to transcription errors and take more of the surgeons' time. However, not all surgeons trust these formulas, having greater experience and satisfactory results with the older ones.

\section{Materials and methods}

Patients. This study included consecutive patients who underwent uncomplicated cataract or refractive lens exchange surgery (88 eyes) with implantation of multifocal IOLs (AcrySof IQ PanOptix TFNT0) at our institution from January 1st, 2018 to December 31st, 2019. 
The study was approved by the Ethics Committee of 'Prof. Dr. Agrippa Ionescu' Emergency Clinical Hospital in Bucharest (Romania) and all patients signed an informed consent after being informed about the benefits and risks of the procedure. The patients were followed-up prospectively at 1 week and 1 month, as scheduled.

Inclusion criteria for surgery were age $\geq 40$, endothelial cell count $>1,500$ cells $/ \mathrm{mm}^{2}$, no corneal opacities, no ocular diseases, no previous ocular surgery or ocular trauma, normal central and peripheral retina, good general health. Exclusion criteria for surgery were patients who did not fulfill the inclusion criteria, irregular astigmatism (10-12), internal silicon oil tamponade, associated retinal pathology (13-22) and poor compliance.

Preoperative assessment. The preoperative ocular examination included: best corrected distance visual acuity (BCVA), manifest refraction, keratometry, tonometry, corneal pachymetry, corneal topography, corneal endothelial cell count, optical coherence biometry, anterior segment slit-lamp biomicroscopy, mydriatic fundoscopy and optical coherence tomography. The refraction, keratometry, non-contact tonometry and optical pachymetry were measured with the autorefracto/kerato/tono/pahimeter Tonoref III (Nidek Co., Ltd.). The corneal endothelial cells were evaluated with the SP 3000P Specular Microscope (Topcon). The optical coherence biometry was measured with the Aladdin HW3.0 (Topcon) (6). This biometer obtains several measures using laser interferometry: axial length (AL), anterior chamber depth, lens thickness, central corneal thickness (6). The keratometry performed with the Aladdin, which scans with Placido disc technology was correlated with the keratometry obtained by the Tonoref III, a device that measures via the double mire ring method. An optical coherence tomography of the macula was performed for each patient with the Cirrus HD-OCT 4000 (Carl Zeiss Meditec AG), in order to rule out potential retinal pathologies that could interfere with the postoperative visual acuity.

Formula calculations. Spherical equivalent formula predictions and lens constant optimizations were performed with the Topcon Aladdin biometer, which has the following on-board calculation formulas: SRKII and SRK/T, Hoffer Q, Holladay 1, Haigis, Barrett Universal II and Olsen IOL formulas for untouched corneas, and Camellin Calossi and Shammas no history, Olsen and Barrett True $\mathrm{K}$ formulas for post refractive surgery IOL calculations.

For this study, the authors focused on the comparison between SRK/T, Hoffer Q, Holladay 1, Haigis and Barrett Universal II. The formulas had the following constants: SRK/T A-constant of 119.100, Hoffer Q pACD of 5.630, Holladay 1 surgeon factor of 1.830 , Haigis' a-constants of 1.390 for $\mathrm{a} 0$, 0.400 for a1 and 0.100 for $\mathrm{a} 2$ and Barrett lens factor of 1.936 . All formulas, including Haigis' a0 constant and Barrett's lens factor were optimized in collaboration with the lens manufacturer for this specific trifocal lens, before starting the study.

The Barrett Formula Suite, Barrett Rx, Barrett Toric Calculator Formula, Barrett True K and Barrett Universal II take into account the posterior cornea, calculate the lens position for each individual patient including consideration of the measured lens thickness rather than an estimation based on patient's age (23). The Barrett Universal II formula predicts IOL power based on Gaussian optics and utilizes this information to calculate the effect of the cylinder power at the cornea (7). Therefore, it needs the following parameters: anterior chamber depth (ACD), AL and keratometry, measured by the optic biometer, as well as 2 constants - Lens Factor and A Constant - available in the biometer software.

The Haigis formula recommends an IOL power based on a three-variable (a0, a1 and a2) function. The a1 constant is tied to the measured ACD, while the a 2 constant is tied to the measured AL. This allows all three constants to be optimized for a wide range of ALs and ACDs using double-regression analysis (24).

Surgical procedure. All surgeries were performed by the same surgeon (H.T.S.) using the same surgical protocol and technique, under local peribulbar anesthesia with $2.5 \mathrm{ml}$ Xiline $4 \%$ and $2.5 \mathrm{ml}$ Marcaine $0.5 \%$. The phacoaspiration (72 eyes)/phacoemulsification (16 eyes) was performed using the INFINITI $^{\circledR}$ Vision System phacoemulsifier (Alcon).

Postoperative treatment and evaluation. Immediately after surgery, the authors prescribed topical eye drops: Moxifloxacin $0.5 \%$, q.d.s. for 1 week, Tobramycin/Dexamethasone $0.3 \% / 0.1 \%$ for 6 weeks (p.d.s. then q.d.s. 1 week each, then t.d.s for 3 weeks), Tropicamide $0.5 \%$ q.d. for 3 weeks and Dexpantenol $5 \%$ gel t.d.s. for 4 weeks.

The first examination was in the first postoperative day, when, after the removal of the bandage, slit lamp examination and mydriatic fundoscopy were performed. The second examination, 1 week after surgery, consisted in the uncorrected distance visual acuity (UDVA), manifest refraction, keratometry, tonometry, corneal pachymetry, anterior segment slit-lamp biomicroscopy and mydriatic fundoscopy.

The third postoperative examination was carried out after a month, when the UDVA, the uncorrected intermediary visual acuity (UIVA), the uncorrected near visual acuity (UNVA), manifest refraction, keratometry, tonometry and pachymetry were measured and also slit-lamp examination was performed and measured. All measurements were performed by the same technician on the same devices, which were calibrated before each measurement.

Data analysis and statistics. Patient data were collected and centralized in an Excel ${ }^{\circledR}$ database (ver. 1902, Microsoft Office 365 ProPlus. Microsoft Corp.) for further analysis. Data analysis was performed on Statistical Package for the Social Sciences (SPSS) software (ver. 24, IBM ${ }^{\circledR}$ SPSS $^{\circledR}$ Statistics, IBM Corp.).

The final postoperative manifest refraction was measured by the same technician, on the same auto-kerato-refractometer 1 month after surgery and it was converted into its spherical equivalent. For the statistical analysis of the postoperative refractive data, the 1-month manifest refraction was analyzed.

All eyes were divided into three groups based on the AL, group 1: <22 mm (14 eyes), group 2: 22-24.5 mm (68 eyes) and group 3: $>24.5 \mathrm{~mm}$ (6 eyes). The refractive prediction error (RPE) and the mean absolute error (MAE) were calculated for 5 different formulas: 3 third generation formulas: SRK-T, Hoffer Q and Holladay 1, and 2 fourth generation formulas: Haigis and Barrett Universal II. 
Table I. Descriptive data of all patients.

\begin{tabular}{lcccc}
\hline Parameter & Age (years) & ACD (mm) & AL (mm) & IOL diopter \\
\hline Mean \pm SD & $62.090 \pm 10.742$ & $3.236 \pm 0.384$ & $23.207 \pm 0.920$ & $22.440 \pm 3.119$ \\
Range & 32 & 1.49 & 3.95 & 15 \\
Minimum & 45 & 2.51 & 21.57 & 17 \\
Maximum & 77 & 4 & 25.50 & 32 \\
\hline
\end{tabular}

ACD, anterior chamber depth; AL, axial length; IOL, intraocular lens.

Table II. Descriptive data of each group.

\begin{tabular}{lcccc}
\hline Parameter & Age (years) & ACD $(\mathrm{mm})$ & AL $(\mathrm{mm})$ & IOL diopter \\
\hline Group 1 & & & & \\
$($ AL $<22 \mathrm{~mm}, \mathrm{n}=14)$ & & & & \\
Mean \pm SD & $65.142 \pm 12.871$ & $2.995 \pm 0.14$ & $21.842 \pm 0.139$ & $26.36 \pm 3.134$ \\
Range & 29 & 0.35 & 0.39 & 8 \\
Minimum & 48 & 2.86 & 21.57 & 32 \\
Maximum & 77 & 3.21 & 21.96 & \\
Group 2 & & & & $22.03 \pm 2.359$ \\
AL 22-24.5 mm, $\mathrm{n}=68)$ & $61.5 \pm 10.453$ & $3.247 \pm 0.392$ & $23.325 \pm 0.612$ & 10 \\
Mean \pm SD & 31 & 1.49 & 2.28 & 17 \\
Range & 45 & 2.51 & 22.09 & 27 \\
Minimum & 76 & 4 & 24.27 & $18 \pm 0.447$ \\
Maximum & & & & 1 \\
Group 3 & & & & 18 \\
(AL $>24.5 \mathrm{~mm}, \mathrm{n}=6)$ & $61.666 \pm 9.048$ & $3.68 \pm 0.226$ & $25.05 \pm 0.431$ & 19 \\
Mean \pm SD & 18 & 0.47 & 0.96 & 24.54 \\
Range & 50 & 3.39 & 25.5 & \\
Minimum & 68 & 3.86 & & \\
Maximum & & & & \\
\hline
\end{tabular}

ACD, anterior chamber depth; AL, axial length; IOL, intraocular lens.

The mean numerical error or RPE represents the difference between the postoperative spherical equivalent at the 1-month follow-up and the predicted postoperative spherical equivalent, chosen by the surgeon from a list generated by the optical coherence biometer (25). A negative predictive error indicates more myopic results, a tendency towards overcorrection, while a positive predictive error indicates more hyperopic results, a tendency towards undercorrection (25). The MAE was calculated as the magnitude of the prediction error, regardless of the sign (25). The proportion of eyes within $\pm 0.25 \mathrm{D}, \pm 0.50 \mathrm{D}$, $\pm 1.00 \mathrm{D}$, and $\pm 2.00 \mathrm{D}$ of the predicted refraction was also calculated in all three groups.

The statistical analysis followed a descriptive stage, which were performed for all cohorts, as well as an inferential stage, which was performed for cohorts with an eye number $>30$. After checking the normality of the distribution of continuous variables by Shapiro-Wilk test, the authors aimed to evaluate the refractive results. The small number of eyes in the first and third group did not allow inferential analysis. The descriptive statistics were reported for each group and outcomes evaluated postoperative for the second group using ANOVA test, analyzing the differences among the five formulas.

The third group of eyes with AL $>24.5 \mathrm{~mm}$ is small, because the authors were still reluctant in using multifocal IOLs, considering the unpredictability factor for myopic eyes, i.e. fundus pathology, ACD variability and capsular bag size with effective lens position instability (26). The first group of eyes with $\mathrm{AL}<22 \mathrm{~mm}$ is also reduced, because in hyperopes, the ideal centration axis for devices that are especially sensitive to position (such as multifocal IOLs) is slightly inferonasal from the optimal location in other eyes, which may also interfere with postoperative visual acuity results (27).

\section{Results}

The study included 88 eyes (44 right eyes and 44 left eyes) from 58 patients ( 38 females and 20 males). Table I displays the descriptive data of all patients. The mean age 
Table III. Mean refractive prediction error, MAE and median absolute error of each formula in each group.

\begin{tabular}{llllll}
\hline Parameter & \multicolumn{1}{c}{ STK-T } & \multicolumn{1}{c}{ Hoffer Q } & Holladay 1 & \multicolumn{1}{c}{ Haigis } & Barrett Universal I \\
\hline Group 1 & & & & & \\
(AL <22 mm, n=14)I & & & & & \\
Mean RPE (D) \pm SD & $0.170 \pm 0.438$ & $-0.108 \pm 0.317$ & $0.120 \pm 0.293$ & $-0.184 \pm 0.526$ & $-0.077 \pm 0.391$ \\
Range RPE & 1.28 & 1.04 & 0.75 & 1.79 & 1.28 \\
MAE (D) \pm SD & $0.332 \pm 0.322$ & $0.262 \pm 0.198$ & $0.220 \pm 0.222$ & $0.370 \pm 0.408$ & $0.297 \pm 0.254$ \\
MedAE & 0.240 & 0.200 & 0.100 & 0.280 & 0.190 \\
Group 2 & & & & \\
(AL 22-24.5mm, n=68) & & & & \\
Mean RPE (D) \pm SD & $0.110 \pm 0.110$ & $0.079 \pm 0.405$ & $0.140 \pm 0.345$ & $0.056 \pm 0.365$ & $0.151 \pm 0.315$ \\
Range RPE & 1.35 & 1.58 & 1.22 & 1.33 & 1.28 \\
MAE (D) \pm SD & $0.322 \pm 0.189$ & $0.327 \pm 0.249$ & $0.302 \pm 0.215$ & $0.300 \pm 0.213$ & $0.292 \pm 0.187$ \\
MedAE & 0.295 & 0.290 & 0.265 & 0.270 & 0.265 \\
Group 3 & & & & & \\
(AL 24.5 mm, n=6) & & & & & $-0.163 \pm 0.201$ \\
Mean RPE (D) \pm SD & $-0.196 \pm 0.100$ & $-0.093 \pm 0.045$ & $-0.176 \pm 0.028$ & $-0.250 \pm 0.062$ & 0.43 \\
Range RPE & 0.22 & 0.10 & 0.06 & 0.13 & $0.223 \pm 0.112$ \\
MAE (D) \pm SD & $0.196 \pm 0.100$ & $0.093 \pm 0.045$ & $0.176 \pm 0.028$ & $0.250 \pm 0.062$ & 0.240 \\
MedAE & 0.170 & 0.100 & 0.190 & 0.220 & \\
\hline
\end{tabular}

RPE, refractive prediction error; MAE, mean absolute error; MedAE, median absolute prediction error; SD, standard deviation.

Table IV. Number of eyes with refractive prediction errors within $\pm 0.25 \mathrm{D}, \pm 0.50 \mathrm{D}, \pm 1.00 \mathrm{D}$, and $\pm 2.00 \mathrm{D}$ in group 1 (AL $<22 \mathrm{~mm}$ ).

\begin{tabular}{lccccc}
\hline & \multicolumn{5}{c}{ Group 1 (n=14) - RPE, no. of eyes } \\
\cline { 2 - 6 } RPE & SRK-T & Hoffer Q & Holladay 1 & Haigis & Barrett Universal II \\
\hline 土.25 D & 4 & 4 & 10 & 6 & 4 \\
$\pm 0.50 \mathrm{D}$ & 12 & 12 & 12 & 8 & 12 \\
$\pm 1.00 \mathrm{D}$ & 12 & 14 & 14 & 14 & 14 \\
$\pm 2.00 \mathrm{D}$ & 14 & 14 & 14 & & 14 \\
\hline
\end{tabular}

RPE, refractive prediction error.

was $62.0 \pm 10.742$ years (from 45 to 77 years). The ACD of the eyes included in the study was $3.236 \pm 0.384 \mathrm{~mm}$ (from 2.51 to $4 \mathrm{~mm}$ ) and the AL was $23.207 \pm 0.920 \mathrm{~mm}$ (from 21.57 to $25.50 \mathrm{~mm}$ ). The Diopter of the AcrySof IQ PanOptix implants ranged from 17 to $32 \mathrm{D}(22.44 \pm 3.119 \mathrm{D})$. Table II presents descriptive data of each group. Table III indicates the mean RPE, MAE, and MedAE (median absolute prediction error) produced by each formula in all three groups. Tables IV-VI indicate the percentage of eyes with RPEs within $\pm 0.25 \mathrm{D}, \pm 0.50 \mathrm{D}, \pm 1.00 \mathrm{D}$, and $\pm 2.00 \mathrm{D}$ in each AL group. In group 2, the greatest percentage of eyes with RPEs within $\pm 0.25 \mathrm{D}$ was $32.4 \%$ for Haigis formula, followed by Barrett Universal II, Hoffer Q and Holladay 1 with $29.4 \%$. However, the percentage of eyes with RPEs within $\pm 0.50 \mathrm{D}$ was $100 \%$ only for Barrett Universal II and Holladay 1, 94.1\% for
SRK/T and 91.2\% for Haigis and Hoffer Q. Group 1 and 3 did not include enough eyes to have any statistical significance. However, it is reported that for group 1, Holladay 1 (10 eyes) had the greatest percentage of eyes RPEs within $\pm 0.25 \mathrm{D}$, followed by Haigis (6 eyes).

Figs. 1-5 show the percentage of eyes with AL between 22 nd $24.5 \mathrm{~mm}$ (group 2) with RPEs (postoperative spherical equivalent minus predicted postoperative spherical equivalent) within the aforementioned diopters and the tendency to overor undercorrect.

In order to analyze the differences between the RPEs of each formula in group 2, which are variables with Gaussian distribution, the parametric test ANOVA was applied. It showed no statistical difference $(\mathrm{P}=0.166)$ between the RPEs measured for each formula in this AL group. 
Table V. Number and percentage of eyes with refractive prediction errors within $\pm 0.25 \mathrm{D}, \pm 0.50 \mathrm{D}, \pm 1.00 \mathrm{D}$, and $\pm 2.00 \mathrm{D}$ in group 2 (AL 22-24.5 mm).

Group 2 (n=68) - RPE, nr. eyes, $\%$

\begin{tabular}{lccccc}
\cline { 2 - 5 } RPE & SRK-T & Hoffer Q & Holladay 1 & Haigis & Barrett Universal II \\
\hline $\pm 0.25 \mathrm{D}$ & $12(17.6)$ & $20(29.4)$ & $20(29.4)$ & $22(32.4)$ & $20(29.4)$ \\
$\pm 0.50 \mathrm{D}$ & $64(94.1)$ & $62(91.2)$ & $68(100)$ & $62(91.2)$ & $68(100)$ \\
$\pm 1.00 \mathrm{D}$ & $68(100)$ & $68(100)$ & $68(100)$ & $68(100)$ & $68(100)$ \\
$\pm 2.00 \mathrm{D}$ & $68(100)$ & $68(100)$ & $68(100)$ & $68(100)$ & $68(100)$
\end{tabular}

RPE, refractive prediction error; AL, axial length.

Table VI. Number of eyes with refractive prediction errors within $\pm 0.25 \mathrm{D}, \pm 0.50 \mathrm{D}, \pm 1.00 \mathrm{D}$, and $\pm 2.00 \mathrm{D}$ in group 3 (AL $>24.5 \mathrm{~mm})$.

Group 3 (n=6)- RPE, nr. eyes

\begin{tabular}{lccccc}
\cline { 2 - 5 } RPE & SRK-T & Hoffer Q & Holladay 1 & Haigis & Barrett Universal II \\
\hline $\pm 0.25 \mathrm{D}$ & 0 & 0 & 0 & 0 & 0 \\
$\pm 0.50 \mathrm{D}$ & 6 & 6 & 6 & 6 & 6 \\
$\pm 1.00 \mathrm{D}$ & 6 & 6 & 6 & 6 & 6 \\
$\pm 2.00 \mathrm{D}$ & 6 & 6 & 6 & 6 & 6 \\
\hline
\end{tabular}

RPE, refractive prediction error; AL, axial length.

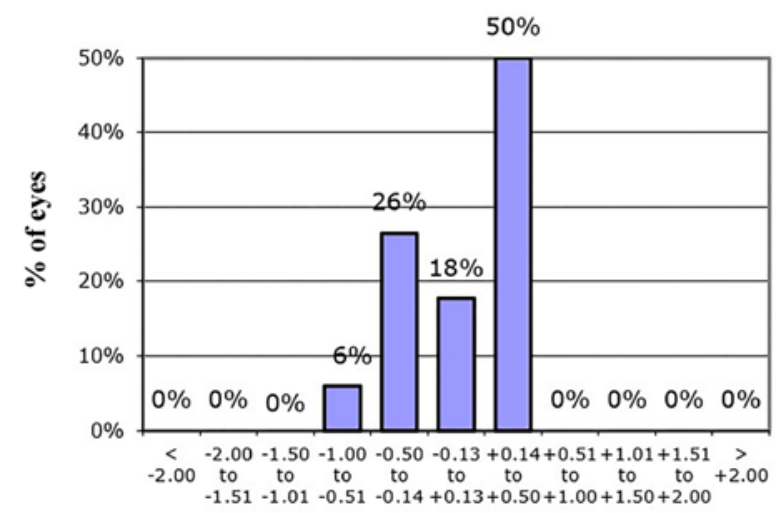

RPE for SRK/T (D)

Figure 1. RPE for SRK/T in group $2(\mathrm{AL}=22-24.5 \mathrm{~mm})$. RPE, refractive prediction error.

\section{Discussion}

With the progress of technology, multifocal IOL implants have become increasingly popular. Patients' expectations for a better refractive outcome and independency of spectacles have grown. In order to meet these expectations, it is important to choose the right lens diopter based on biometric formulas. Alcon Acrysof IQ PanOptix lenses have been on the European market since 2015 (26) and have recently been approved by the FDA as the first trifocal lens for USA patients undergoing

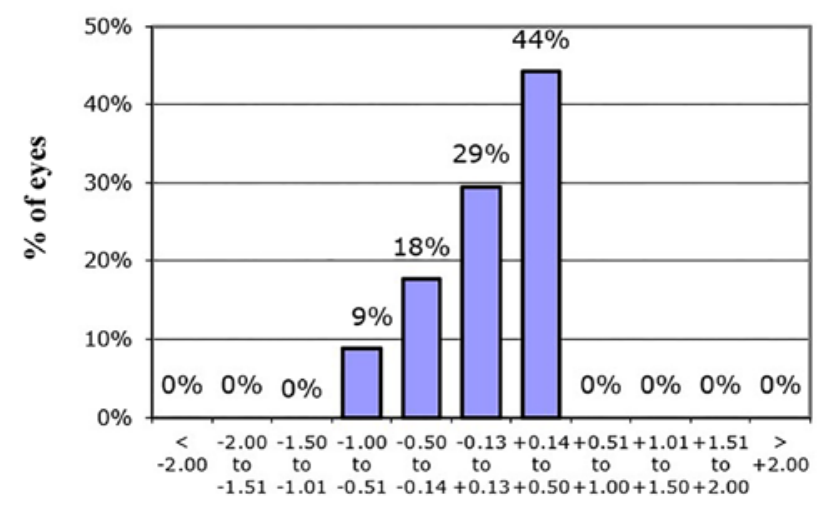

RPE for Hoffer Q (D)

Figure 2. RPE for Hoffer Q in group $2(\mathrm{AL}=22-24.5 \mathrm{~mm})$. RPE, refractive prediction error.

cataract surgery (2019) (27). The accuracy of each biometric formula for this type of lens should be assessed in more studies in order to avoid refractive surprises, given the fact that both patients' expectations and lens fabrication prices are higher.

This study shows no statistical difference between formulas for this multifocal lens implant in the AL group between 22 and $24.5 \mathrm{~mm}$. There is, however, a tendency for the RPE to be within $\pm 0.25 \mathrm{D}$ for most of the eyes with the Haigis formula, and within $\pm 0.50 \mathrm{D}$ for all the eyes with the Barrett Universal II and Holladay 1 formula in this group. For 


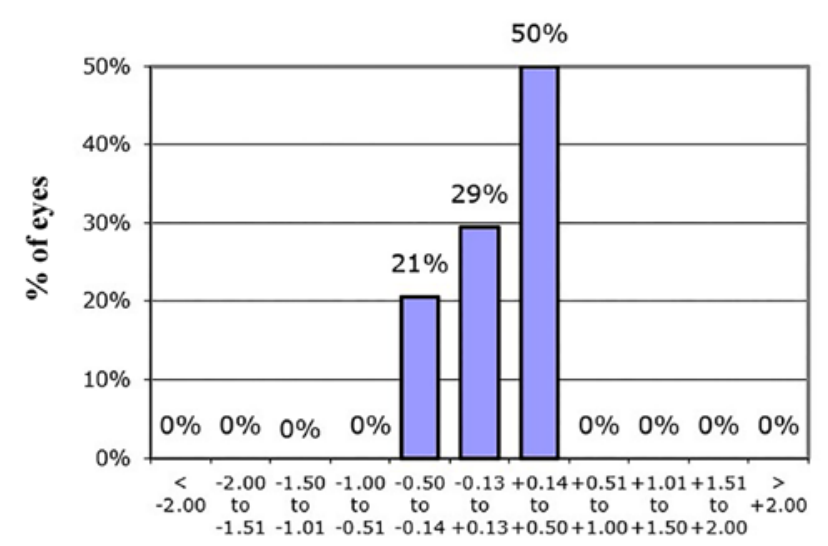

RPE for Holladay 1 (D)

Figure 3. RPE for Holladay 1 in group $2(\mathrm{AL}=22-24.5 \mathrm{~mm})$. RPE, refractive prediction error.

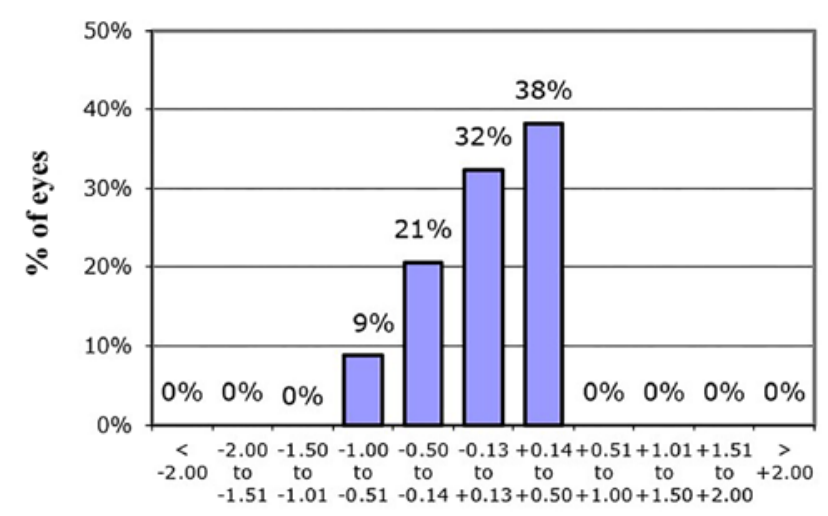

RPE for Haigis (D)

Figure 4. RPE for Haigis in group $2(\mathrm{AL}=22-24.5 \mathrm{~mm})$. RPE, refractive prediction error.

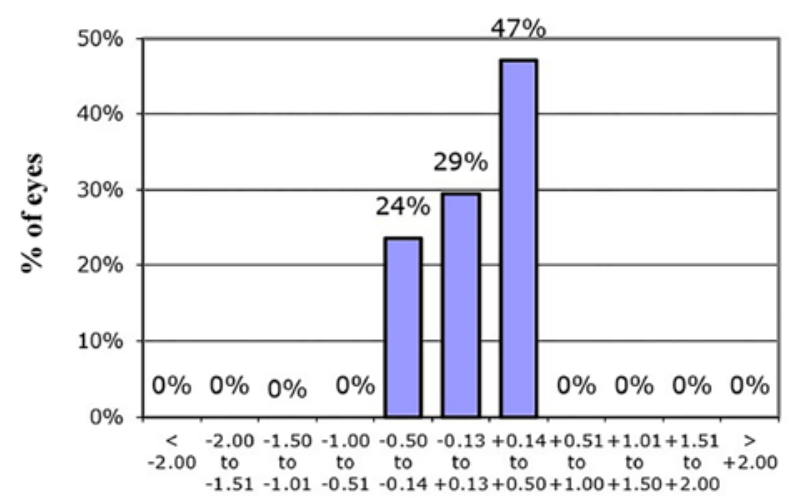

RPE for Barrett Universal II (D)

Figure 5. RPE for Barrett Universal II in group 2 ( $\mathrm{AL}=22-24.5 \mathrm{~mm})$. RPE, refractive prediction error.

long and short eyes, data on more surgeries should be collected in order to obtain statistically significant results.

For myopic eyes, Zhu et al (28) showed greater inferior decentration of multifocal IOLs (Tecnis ZMB00), indicating that the increasing incompatibility between IOL and capsular bag size with AL elongation should not be underestimated. For hyperopic eyes, the ideal centration axis in devices that are especially sensitive to position (such as multifocal IOLs) is slightly inferonasal compared with the optimal location in other eyes $(15,29)$. However, multifocal lenses should be centered as closely as possible on the entrance pupil because they need balanced light input to create two focal points via constructive interference of the diffracted light (29).

There are some studies reporting good visual outcomes in patients with Acrysof IQ PanOptix trifocal implants. Belikova and Borzykh published a study evaluating patient spectacle independence and high-quality of vision in mesopic conditions after bilateral implantation of AcrySof IQ PanOptix in 16 patients, indicating good results (30). Sezgin Asena compared the clinical performance of a hydrophobic (AcrySof PanOptix) and a hydrophilic (AT LISA tri 839MP) diffractive trifocal IOL using the SRK/T (AL >22.0 mm) or Hoffer Q formula $(\mathrm{AL}<22.0 \mathrm{~mm})$, concluding that hydrophobic diffractive IOL might be more suitable for patients who require good closer intermediate viewing (31). Alió et al also reported that the AcrySof IQ Panoptix IOL is able to restore visual function with an acceptable intermediate and near vision after cataract surgery with good contrast sensitivity and an improvement in the near activity visual questionnaire (32).

Regarding target refraction in eyes with this type of lenses, Hayashi et al published a study suggesting that slight myopia is a better target refraction than slight hyperopia, although emmetropia is the optimum target (33). In this study, the minus diopter closest to zero was also targeted, and the postoperative spherical equivalent to it was compared, in order to obtain the RPE for each formula.

Cochener et al compared the performance of two diffractive trifocal (one of which was PanOptix) and one extended depth of focus hydrophobic (EDOF) IOLs using Haigis formula. The study concluded that near vision was statistically better for both trifocal IOLs compared with the EDOF IOL (34).

However, none of these studies focused on which formula is best when choosing the right diopter for the trifocal lens. Comparing the accuracy of the Barrett Universal II formula, several studies concluded that this formula is well suited in calculating IOL power for all types of eyes (1-3), including those with high myopia $(35,36)$ rendering the lowest predictive error compared with SRK/T, Haigis, Holladay 1, and Hoffer Q formulas. The performances of the Barrett Universal II and Hill-RBF formulas were proven comparable in long eyes in two studies evaluating the accuracy of new generation vergence formulas and formulas based on artificial intelligence $(37,38)$.

Regarding short eyes, studies have shown Haigis' formula superiority over second generation Hoffer Q, considering that Haigis takes ACD into account when calculating the IOL power $(39,40)$. The differences between the predicted refractive errors of the Hoffer Q and Haigis formula increased as ACD decreased in hyperopic eyes (39).

The fact that Haigis showed the smallest RPE in our study might be explained by the fact that the formula constants were optimized regularly in collaboration with the lens manufacturers, which obtained data from databases containing all the recent cases. The Barrett Universal II formula, which showed the greatest results in the most recent studies $(1,3,35,36)$ is newer, and not yet widely used, which may affect the 
optimization process. Nevertheless, it did come in second place for this group. The ANOVA test, however, showed no statistical difference between all the evaluated formulas, which means that for this cohort there was no inferiority between formulas.

This study represents only a first stage in a wider evaluation of biometric formulas and refractive postoperative results. It will be expanded by collecting many more cases of trifocal IOL implants from all three AL categories and it will statistically revaluate groups 1 and 3 , after they will exceed 30 cases.

It is important to assess the efficacy of the biometric formulas for each type of lens, especially for multifocal lenses, considering the rise in patients' expectations and demand for this type of implants. To our knowledge, there is no other study comparing the efficacy of formulas for a trifocal lens.

\section{Acknowledgements}

Professional editing, linguistic and technical assistance performed by Irina Radu, Individual Service Provider, certified translator in Medicine and Pharmacy (certificate credentials: Series E no. 0048).

\section{Funding}

No funding was received.

\section{Availability of data and materials}

All data generated or analyzed during this study are included in this published article.

\section{Authors' contributions}

MM and HTS contributed to the conception and design of the study, and the acquisition, analysis and interpretation of the data. MM and HTS also contributed to the drafting of the manuscript and its critical revision for important intellectual content. SS contributed to the design of the study, the drafting of the manuscript and its critical revision for important intellectual content. BT contributed to the acquisition, analysis and interpretation of the data, the drafting of the manuscript and its critical revision for important intellectual content. AS contributed to the analysis and interpretation of the data, the drafting of the manuscript and its critical revision for important intellectual content. $\mathrm{CD}$ contributed to the conception and design of the study and the critical revision of the manuscript for important intellectual content. All authors read and approved the final version of the manuscript and agreed to be accountable for all aspects of the study in ensuring that questions related to the accuracy or integrity of any part of the work are appropriately investigated and resolved.

\section{Ethics approval and consent to participate}

The study was approved by the Ethics Committee of 'Prof. Dr. Agrippa Ionescu' Emergency Clinical Hospital in Bucharest, Romania. All patients provided a signed informed consent.

\section{Patient consent for publication}

Not applicable.

\section{Competing interests}

The authors declare that they have no competing interests.

\section{References}

1. Kane JX, Van Heerden A, Atik A and Petsoglou C: Intraocular lens power formula accuracy: Comparison of 7 formulas. J Cataract Refract Surg 42: 1490-1500, 2016.

2. Cooke DL and Cooke TL: Comparison of 9 intraocular lens power calculation formulas. J Cataract Refract Surg 42: 1157-1164, 2016.

3. Melles RB, Holladay JT and Chang WJ: Accuracy of intraocular lens calculation formulas. Ophthalmology 125: 169-178, 2018.

4. Kohnen T: First implantation of a diffractive quadrafocal (trifocal) intraocular lens. J Cataract Refract Surg 41: 2330-2332, 2015.

5. Sudhir RR, Dey A, Bhattacharrya S and Bahulayan A: AcrySof IQ PanOptix intraocular lens versus extended depth of focus intraocular lens and trifocal intraocular lens: A clinical overview. Asia Pac J Ophthalmol (Phila) 8: 335-349, 2019.

6. Mandal P, Berrow EJ, Naroo SA, Wolffsohn JS, Uthoff D, Holland D and Shah Sunil: Validity and repeatability of the Aladdin ocular biometer. Br J Ophthalmo 98:256-258, 2014.

7. Haigis W, Lege B, Miller N and Schneider B: Comparison of immersion ultrasound biometry and partial coherence interferometry for intraocular lens calculation according to Haigis. Graefes Arch Clin Exp Ophthalmol 238: 765-773, 2000.

8. Weeber HA, Meijer ST and Piers PA: Extending the range of vision using diffractive intraocular lens technology. J Cataract Refract Surg 41: 2746-2754, 2015.

9. Lawless M, Hodge C, Reich J, Levitz L, Bhatt UK, McAlinden C, Roberts $\mathrm{K}$ and Roberts TV: Visual and refractive outcomes following implantation of a new trifocal intraocular lens. Eye Vis (Lond) 4: 10, 2017.

10. Koch DD, Ali SF, Weikert MP, Shirayama M, Jenkins R and Wang L: Contribution of posterior corneal astigmatism to total corneal astigmatism. J Cataract Refract Surg 38: 2080-2087, 2012.

11. Stanca HT, Munteanu M, Jianu DC, Motoc AGM, Jecan CR, Tăbăcaru B, Stanca S and Preda MA: Femtosecond-LASIK outcomes using the VisuMax ${ }^{\circledR}-$ MEL $^{\circledR} 80$ platform for mixed astigmatism refractive surgery. Rom J Morphol Embryol 59: 277-283, 2018.

12. Hayashi K, Manabe S, Yoshida M and Hayashi H: Effect of astigmatism on visual acuity in eyes with a diffractive multifocal intraocular lens. J Cataract Refract Surg 36: 1323-1329, 2010.

13. Soda M and Yaguchi S: Effect of decentration on the optical performance in multifocal intraocular lenses. Ophthalmologica 227: 197-204, 2012.

14. Braga-Mele R, Chang D, Dewey S, Foster G, Henderson BA, Hill W, Hoffman R, Little B, Mamalis N, Oetting T, et al; ASCRS Cataract Clinical Committee: Multifocal intraocular lenses: Relative indications and contraindications for implantation. J Cataract Refract Surg 40: 313-322, 2014.

15. Walkow T, Anders N, Pham DT and Wollensak J: Causes of severe decentration and subluxation of intraocular lenses. Graefes Arch Clin Exp Ophthalmol 236: 9-12, 1998.

16. Stanca HT, Suvac E, Munteanu M, Jianu DC, Motoc AGM, Roşca GC and Boruga O: Giant cell arteritis with arteritic anterior ischemic optic neuropathy. Rom J Morphol Embryol 58: 281-285, 2017.

17. Stanca HT, Petrović Z and Munteanu M: Transluminal Nd:YAG laser embolysis - a reasonable method to reperfuse occluded branch retinal arteries. Vojnosanit Pregl 71: 1072-1077, 2014.

18. Stratton IM, Kohner EM, Aldington SJ, Turner RC, Holman RR, Manley SE and Matthews DR: UKPDS 50: Risk factors for incidence and progression of retinopathy in Type II diabetes over 6 years from diagnosis. Diabetologia 44: 156-163, 2001.

19. Verrotti A, Lobefalo L, Petitti MT, Mastropasqua L, Morgese G, Chiarelli $\mathrm{F}$ and Gallenga PE: Relationship between contrast sensitivity and metabolic control in diabetics with and without retinopathy. Ann Med 30: 369-374, 1998. 
20. Stanca HT, Stanca S, Tabacaru B, Boruga M and Balta F. Bevacizumab in Wet AMD treatment: A tribute to the thirteen years of experience from the beginning of the anti-VEGF era in Romania. Exp Ther Med 18: 4993-5000, 2019.

21. Kleiner RC, Enger C, Alexander MF and Fine SL: Contrast sensitivity in age-related macular degeneration. Arch Ophthalmol 106: 55-57, 1988.

22. Chew EY, Sperduto RD, Milton RC, Clemons TE, Gensler GR, Bressler SB, Klein R, Klein BEK and Ferris FL III: Risk of advanced age-related macular degeneration after cataract surgery in the Age-Related Eye Disease Study: AREDS report 25. Ophthalmology 116: 297-303, 2009.

23. Hoffer KJ: The Hoffer Q formula: A comparison of theoretic and regression formulas. J Cataract Refract Surg 19: 700-712, 1993.

24. Hoffer KJ, Aramberri J, Haigis W, Olsen T, Savini G, Shammas HJ and Bentow S: Protocols for studies of intraocular lens formula accuracy. Am J Ophthalmol 160: 403-405, 2015.

25. Shrivastava AK, Behera P, Kacher R and Kumar B: Effect of anterior chamber depth on predictive accuracy of seven intraocular lens formulas in eyes with axial length less than $22 \mathrm{~mm}$. Clin Ophthalmol 13: 1579-1586, 2019.

26. American Academy of Ophthalmology. New trifocal IOL receives CE mark in Europe. The Ophthalmic News and Education Network. 2015 Jun 17. Accessed March 31, 2020. https://www. aao.org/headline/new-trifocal-iol-receives-ce-mark-in-europe.

27. Delfaro A: Alcon's trifocal IOL makes U.S. debut. American Academy of Ophthalmology. The Ophthalmic News and Education Network. Accessed 2020, March 31. https://www.aao. org/headline/alcon-s-trifocal-iol-makes-u-s-debut.

28. Zhu X, He W, Zhang Y, Chen M, Du Y and Lu Y: Inferior decentration of multifocal intraocular lenses in myopic eyes. Am J Ophthalmol 188: 1-8, 2018.

29. Roach L: Centration of IOLs: Challenges, Variables, and Advice for Optimal Outcomes. EyeNet Magazine / April 2013. Accessed March 31, 2020. https://www.aao.org/eyenet/article/centrationof-iols-challenges-variables-advice-opt?april-2013.

30. Belikova EI and Borzykh VA: Results of trifocal intraocular lenses implantation in patients with cataract and presbyopia. Ophthalmol Russ 15: 248-255, 2018 (In Russian).

31. Sezgin Asena B: Visual and refractive outcomes, spectacle independence, and visual disturbances after cataract or refractive lens exchange surgery: Comparison of 2 trifocal intraocular lenses. J Cataract Refract Surg 45: 1539-1546, 2019.
32. Alió JL, Plaza-Puche AB, Alió Del Barrio JL, Amat-Peral P, Ortuño V, Yébana P, Al-Shymali O and Vega-Estrada A: Clinical outcomes with a diffractive trifocal intraocular lens. Eur J Ophthalmol 28: 419-424, 2018.

33. Hayashi K, Sato T, Igarashi C and Yoshida M: Effect of spherical equivalent error on visual acuity at various distances in eyes with a trifocal intraocular lens. J Refract Surg 35: 274-279, 2019.

34. Cochener B, Boutillier G, Lamard M and Auberger-Zagnoli C: A comparative evaluation of a new generation of diffractive trifocal and extended depth of focus intraocular lenses. J Refract Surg 34: 507-514, 2018.

35. Rong X, He W, Zhu Q, Qian D, Lu Y and Zhu X: Intraocular lens power calculation in eyes with extreme myopia: Comparison of Barrett Universal II, Haigis, and Olsen formulas. J Cataract Refract Surg 45: 732-737, 2019.

36. Zhou D, Sun Z and Deng G: Accuracy of the refractive prediction determined by intraocular lens power calculation formulas in high myopia. Indian J Ophthalmol 67: 484-489, 2019.

37. Wan KH, Lam TCH, Yu MCY and Chan TCY: Accuracy and precision of intraocular lens calculations using the new Hill-RBF Version 2.0 in eyes with high axial myopia. Am J Ophthalmol 205: 66-73, 2019.

38. Liu J, Wang L, Chai F, Han Y, Qian S, Koch DD and Weikert MP. Comparison of intraocular lens power calculation formulas in Chinese eyes with axial myopia. J Cataract Refract Surg 45: 725-731, 2019.

39. Eom Y, Kang SY, Song JS, Kim YY and Kim HM: Comparison of Hoffer Q and Haigis formulae for intraocular lens power calculation according to the anterior chamber depth in short eyes. Am J Ophthalmol 157: 818-824.e2, 2014.

40. MacLaren RE, Natkunarajah M, Riaz Y, Bourne RR, Restori M and Allan BD: Biometry and formula accuracy with intraocular lenses used for cataract surgery in extreme hyperopia. Am J Ophthalmol 143: 920-931, 2007.

This work is licensed under a Creative Commons Attribution-NonCommercial-NoDerivatives 4.0 International (CC BY-NC-ND 4.0) License. 